Proceedings of the 9th National Symposium of Synchrotron Radiation Users, Warsaw, September 26-27, 2011

\title{
Status and Vision for Structural Biology at the Canadian Light Source
}

\author{
P. Grochulski ${ }^{a, b *}$, M.N. Fodje $^{a, c}$ ANd G. GeOrGE ${ }^{d}$ \\ ${ }^{a}$ Canadian Light Source, 101 Perimeter Road, Saskatoon, SK S7N 0X4 Canada \\ ${ }^{b}$ College of Pharmacy and Nutrition, University of Saskatchewan, 110 Science Place, \\ Saskatoon, SK S7N 5C8, Canada
}

${ }^{c}$ Department of Biochemistry, University of Saskatchewan, 107 Wiggins Road, Saskatoon SK S7N 5E5, Canada ${ }^{d}$ Department of Geology, University of Saskatchewan, 114 Science Place, Saskatoon, SK S7N 5E2, Canada

The status and vision for Structural Biology at the Canadian Light Source (CLS) is presented. The beamlines that have been described in the paper represent a Canadian national resource that is available to science and industry world-wide. They include state-of-the-art infrastructure and include specialized capabilities, many of which are not available elsewhere, including macromolecular crystallography, biological X-ray spectroscopy, soft X-ray spectromicroscopy, as well as small angle and wide angle X-ray scattering beamlines. The vision for Structural Biology at the Canadian Light Source is significantly enhanced by the synergies and collaborations between the users of the different beamlines and by the strengths of the scientific personnel and trainees.

PACS: 87.64.kd, 87.80.Dj

\section{Introduction}

The Canadian Light Source (CLS) is a $2.9 \mathrm{GeV}$ national synchrotron radiation facility located at the University of Saskatchewan in Saskatoon. The Canadian Macromolecular Crystallography Facility (CMCF) is located at the CLS and consists of the 08ID-1 and 08B11 beamlines. The CMCF, together with the Biological X-ray Absorption Spectroscopy (BioXAS) and soft Xray spectromicroscopy (SM) beamlines, form a suite of beamlines that allow Canadian researchers to conduct cutting-edge research in structural biology at the CLS. This suite will soon be complemented with small- and wide-angle X-ray scattering (SAXS/WAXS) capabilities.

The number of beamlines worldwide that contribute to the field of structural biology is growing and the current statistics for mid-energy rings are outlined in Table I. The beamlines included in the Table comprise macromolecular crystallography (MX), biological XAS and biological SAXS/WAXS beamlines. Some of the biological SAXS beamlines serve material science as well. The growing need for biological SAXS beamlines is demonstrated by the magnitude and sophistication of the SAXS beamline being built at the PetraIII facility in Hamburg, Germany. At all facilities mentioned in Table I, the number of MX beamlines surpasses that of other techniques, indicating that a large number of researchers require high resolution structures of macromolecules to solve their biological problems. Productivity of the MX beamlines is very easy to monitor due to the BioSync Web initiative, where information is tabulated from over $130 \mathrm{MX}$

\footnotetext{
* e-mail: pawel.grochulski@lightsource.ca
}

TABLE I

2.5-3.0 GeV synchrotron facilities worldwide and the number of MX, BioXAS and BioSAXS/WAXS beamlines

\begin{tabular}{l|c|c|c}
\hline \hline \multirow{2}{*}{ Synchrotron facility } & MX & BioXAS $^{\dagger}$ & BioSAXS/WAXS \\
\cline { 2 - 4 } & \multicolumn{3}{|c}{ number of beamlines } \\
\hline CLS (CA) & 2 & $3^{*}$ & $1^{* *}$ \\
SLS (CH) & 3 & 0 & 1 \\
SSRL (USA) & 5 & 4 & 1 \\
ALS (USA) & 8 & 0 & $1^{\#}$ \\
Diamond (GB) & $5+1^{*}$ & 0 & $1^{*}$ \\
Solei (F) & 2 & 0 & 1 \\
Australian & 2 & 1 & 1
\end{tabular}

*indicates beamlines being built, **indicates beamlines in the planning stage, ${ }^{\dagger}$ defined as beamlines specifically designed for BioXAS, or for which BioXAS forms a large fraction of the research, ${ }^{\ddagger}$ defined as beamlines designed for BioSAXS or for which BioSAXS forms large fraction of research, " that beamline is split about $50 / 50$ between MX and SAXS.

beamlines at synchrotron radiation facilities worldwide [1]. For example, the CMCF 08ID-1 beamline has been performing very well, having had 94 deposited structures in 2010 and 57 in 2011. At this time, the other synchrotron techniques described in this paper do not have a similar formal way of tracking productivity.

\section{Experimental Facilities}

\subsection{Canadian Macromolecular Crystallography Facility}

The CMCF consists of the 08ID-1 and 08B1-1 beamlines and provides service to more than 60 Principal Investigators in Canada and the United States [2]. Up to $25 \%$ of the beam time is devoted to commercial users and the general user program is guaranteed up to $55 \%$ of the useful beam time which is administered under a peerreview proposal system. There are two modes of access to 
the CMCF; remote access and on-site data acquisition. In the remote mode, users either control the beamline remotely from their home facilities, or the CMCF staff provide "Mail-In" crystallography service. Users with the highest scored proposals are given priority for the "MailIn" service.

TABLE II

Specifications of the CMCF beamlines

\begin{tabular}{|c|c|c|}
\hline & 08ID-1 & 08B1-1 \\
\hline Spectral range (keV) & $6.0-18.0$ & $4.0-18.0$ \\
\hline$(\AA)$ & $2.1-0.7)$ & $3.1-0.7$ \\
\hline $\begin{array}{lr}\text { Energy } & \text { bandwidth } \\
(\Delta E / E) & \mathrm{Si}(111) @ 12 \\
\mathrm{keV} & \end{array}$ & $1.5 \times 10^{-4}$ & $1.4 \times 10^{-4}$ \\
\hline $\begin{array}{l}\text { Measured Focal Size } \\
@ 12 \mathrm{keV}(\mathrm{FWHM}) \\
(\mathrm{H} \mu \mathrm{m} \times \mathrm{V} \mu \mathrm{m})\end{array}$ & $150 \times 50$ & $210 \times 190$ \\
\hline $\begin{array}{l}\text { Flux on the sample @ } \\
12 \mathrm{keV}(250 \mathrm{~mA}) \text { (pho- } \\
\text { tons } / \mathrm{s} \text { ) (from } 6 \text { th har- } \\
\text { monic of the ID) }\end{array}$ & $\begin{array}{l}5 \times 10^{12} \\
2 \times 10^{12}(100 \mu \mathrm{m}), \\
1 \times 10^{12}(50 \mu \mathrm{m}), \\
7 \times 10^{11}(20 \mu \mathrm{m}) \\
2 \times 10^{10}(5 \mu \mathrm{m})\end{array}$ & $1.0 \times 10^{11}$ \\
\hline Typical beam size & $100 \mu \mathrm{m}$ & $200 \mu \mathrm{m}$ \\
\hline $\begin{array}{l}\text { Beam crossfire at } \\
\text { the sample @ } \\
12 \quad \mathrm{keV}(\mathrm{FWHM}) \\
(\mathrm{H} \text { mrad } \times \mathrm{V} \text { mrad })\end{array}$ & $0.9 \times 0.2$ & $1.8 \times 0.3$ \\
\hline
\end{tabular}

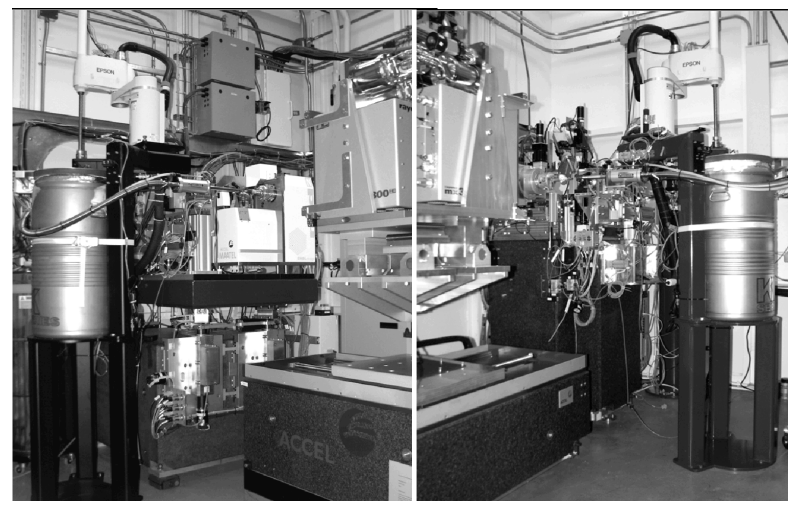

Fig. 1. Overall view of the $\mathrm{CMCF}$ endstations; on the left panel of the $08 \mathrm{~B} 1-1$ beamline and on the right panel of the 08ID-1 beamline.

Beamline 08ID-1 is an undulator-based beamline capable of measuring data from small crystals and crystals with large cell dimensions. The 08B1-1 beamline is a bend magnet-based beamline which is fully automated and equipped for remote access. The specifications of the CMCF beamlines are shown in Table II and end stations are shown in Fig. 1. The $08 \mathrm{ID}-1$ beamline is a very bright beamline allowing selection of beam sizes as small as $5 \mu \mathrm{m}$ with flux of $2 \times 10^{10}$ photons/s. However the typical beam size used at this beamline is $100 \mu \mathrm{m}$, delivering a flux on the sample of $2 \times 10^{12}$ photons/s (Table II). The 08B11 beamline is equipped with a mini-Kappa goniometer

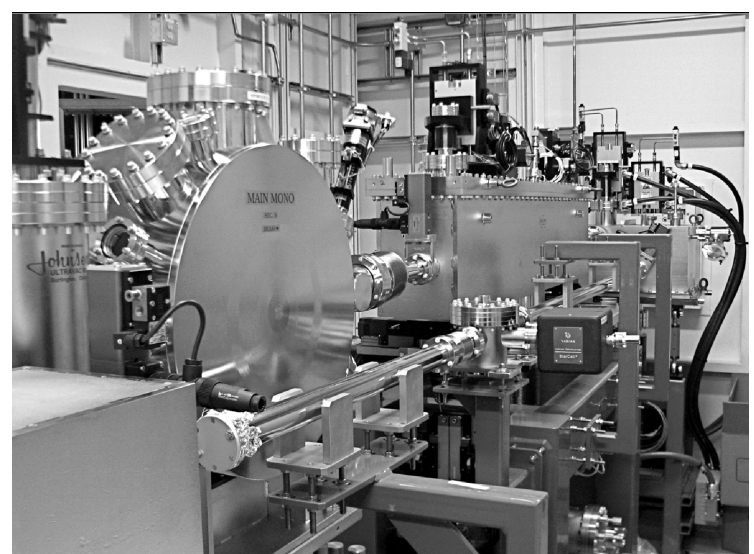

Fig. 2. Picture of the optical components of the BioXAS beamlines.

and a VORTEX-4M detector, allowing performance of EXAFS experiments on protein crystals. On request, either beamline can be equipped with a MAATEL HC1 humidity control device for optimizing diffraction from difficult and poorly-diffracting crystals. The beamlines are equipped with very robust end-stations including onaxis visualization systems, Rayonix 300 series CCD detectors and Stanford-type robotic sample automounters (SAM) allowing remote control of the beamlines. The SAM robot accepts Uni-Pucks and/or SSRL-style cassettes. Depending on the configuration it can hold up to 192 crystals.

As with all facilities at the CLS, the low-level control system of the beamlines is based on the Experimental Physics and Industrial Control System (EPICS). MxDC, the in-house developed data acquisition software, has an integrated and user-friendly graphical user interface with features for performing beamline setup, automated sample mounting and alignment, fluorescence scans for identifying metals in protein crystals or selecting wavelengths for MAD data collection. MxDC is integrated with a data processing module, AutoProcess, allowing full automation of data collection and data processing with minimal human intervention $[3,4]$. It also allows remote control and monitoring of experiments through interaction with MxLIVE, a MX Laboratory Information Virtual Environment [3]. This CMCF Laboratory Information Management System was also developed at the facility with features for managing and conveying sample information to the facility, planning of experiments to be performed on samples, inspecting and downloading experimental results, all from within a web browser. The data processing module, known as AutoProcess, is a data processing pipeline based on the XDS [5], Pointless [6], CCP4 [7], BEST [8] and XDSSTAT [9] packages. AutoProcess allows automatic calculation of optimal strategies for data collection and automatic processing of native, anomalous and MAD datasets with minimal user input.

The number of scientific papers for which data were collected at the 08ID-1 beamline exceeds 100 at present, 
including such prestigious journals as Science, Nature, PNAS, J. Mol. Biol. and Cell, the number of PDB entries exceeding 220 structures. A very recent example of science from the CMCF is the determination of the crystal structures of the TgAMA1 apo form and complex formed with a RON2 peptide at $2.25 \AA$ and $1.95 \AA$ resolution, respectively [10]. Toxoplasma and Plasmodium parasites cause numerous diseases worldwide, including malaria and toxoplasmosis. These parasites attack host cells in a very active manner, providing the receptor for binding to the host cell. Interaction thus occurs through a protein called AMA1 to a rhoptry neck (RON) complex provided by the parasite and injected into the host cell. Besides providing insights into host cell invasion by apicomplexan parasites, the structure offers a basis for designing therapeutics targeting these global pathogens.

\subsection{Future development}

In the genomics era, crystals are getting smaller and smaller, frequently with dimensions less than $10 \mu \mathrm{m}$. To be able to collect data from such small crystals the $\mathrm{Hu}-$ ber single axis goniometer of the 08ID-1 beamline will be upgraded to an air-bearing with a sphere of confusion of less than $1 \mu \mathrm{m}$. That implementation will allow proper utilization of the mini-beam apparatus to deliver a $5 \mu \mathrm{m}$ beam on a $5 \mu \mathrm{m}$ crystal. To expand the capabilities of the CMCF we are planning to add other spectroscopic techniques. For example, Raman, EXAFS and UV-Visible absorption spectroscopies can provide information that compliments X-ray diffraction data. The 08B1-1 beamline will therefore be upgraded by enhancing the X-rayabsorption spectroscopy (EXAFS) capabilities with additional in situ spectroscopic methods such as UV-Visible absorption and possibly Raman spectroscopies.

\subsection{Biological X-ray Absorption Spectroscopy} (BioXAS)

X-ray spectroscopy (XAS) contributes to structural biology in two ways. It can provide information on species for which crystal structures are not available and it can also provide supplemental information on systems for which crystal structures are available. Since about $30 \%$ of the human genome is made up of genes encoding metalloproteins, XAS, but in particular the extended X-ray absorption fine structure (EXAFS), is extremely useful when studying the coordination environment of biological metal ions within a radius of $5 \AA$. Its major strength is that very accurate values for average bond lengths can be obtained, typically to better than $\pm 0.02 \AA$, which is comparable to accuracies obtained for small-molecule structures [11], although precisions are typically about an order of magnitude better than this and relative changes in similar species can be very well defined. The EXAFS accuracy is about ten times better than precisions obtained from a typical protein crystallography experiment at a moderate resolution [12] and this may be important in understanding the detailed chemistry of metal ion coordination within a biological system. The EXAFS bond-length resolution, $\Delta R$, is defined as the minimum distance between similar ligands that can be discerned from analysis of a data set, and this is directly related to the extent of the data and is approximately given by $\Delta R \approx \pi / 2 k$, where $k$ is the photo-electron wave vector and the extent of the data, typically measured in $\AA^{-1}$. For typical EXAFS data ranges, $\Delta R$ is rather poor at around $0.15 \AA$, and this is one major limitation of EXAFS analysis [12]. In some cases this limitation can be overcome by extending the data range. While simple in principle, collecting EXAFS data at high- $k$ requires very stable beamline optics, an excellent signal to noise ratio, and sample limitations in that no elements whose absorption edges might truncate the $k$-range can be present. EXAFS can also determine coordination numbers of similarly coordinated ligands, $N$, and estimates of the Debye-Waller factors, $\sigma^{2}$, a measure of the mean-square-deviation in average bond-length $R$. Both $N$ and $\sigma^{2}$ are typically determined with an accuracy of around $\pm 25 \%$. In most cases no direct information on geometry (e.g. bond-angles) is available from EXAFS, but in some experiments significant multiple scattering (MS) is observed. In this case the EXAFS can be sensitive to the relative arrangement of the atoms providing limited information on bond angles.

The BioXAS facility at the CLS has been designed, all major components have been purchased and it is currently under construction. The facility will be composed of three beamlines powered by two different insertion devices, allowing the study of biological and health-related metals. The two spectroscopy BioXAS lines both have an energy range of $5-28 \mathrm{keV}$ (including the K-edges of $\mathrm{V}$ to $\mathrm{Cd}$, and the L-edges of $\mathrm{Cs}$ and elements above). The undulator endstation (BioXAS-I) will also go slightly lower - to $4 \mathrm{keV}$ which will allow access to the K-edge of Calcium and up. The optical hutch of the BioXAS beamlines is shown in Fig. 2. Two of the three beamlines will be optimized for XAS measurements and powered by a high-field flat-top field profile permanent magnet wiggler. These beamlines will provide a stable platform for EXAFS studies of metals in biological molecules, with a number of multi-element solid state detectors to allow studies of dilute systems. The third beamline is an imaging endstation with micro-focus capability that will allow micro-XAS on very small regions and will be powered by an in-vacuum undulator. The imaging end station will be capable of imaging biological samples by monitoring $\mathrm{X}$-ray fluorescence with three different resolution ranges; macro with a 50-150 $\mu \mathrm{m}$ beam size for large samples (up to $600 \mathrm{~mm}$ across), micro for smaller samples with a 3-5 $\mu \mathrm{m}$ beam size, and nano with $150-250 \mathrm{~nm}$ beam size. The BioXAS imaging beamline will have the unique capability of being able to rapidly change between the different resolutions available.

Metal ions are important for some of the most complex chemistry carried out by living organisms, and, for example, are essential components of the limiting enzymes of the carbon, nitrogen and oxygen cycles. Apart from this fundamental importance, metal ions are also important in numerous diseases. Examples include progressive neurodegenerative diseases such as Alzheimer's 
disease, Parkinson's disease [13] and multiple sclerosis, diseases of metal dysregulation such as Wilson's [14] and Menke's diseases, and severe fatal complaints such as sulfite oxidase deficiency. Metal ions are also important environmental toxins (e.g. mercury and arsenic), and there is also growing interest in new metal-containing pharmaceuticals, such as platinum and ruthenium-based chemotherapeutic drugs. The BioXAS infrastructure at CLS will provide world-class capabilities for research in all of these fields, and will be highly complementary to the other life-science cluster beamlines.

\subsection{Soft X-ray spectromicroscopy}

The CLS soft X-ray spectromicroscopy (SM) beamline combines X-ray absorption spectroscopy in the $130 \mathrm{eV}$ to $2.5 \mathrm{keV}$ energy range with microscopy (2-D or $3-\mathrm{D})$ at a high spatial resolution of $25-30 \mathrm{~nm}$ [15]. It is used to study the interactions of proteins, lipids, and metals and to map their distributions. The samples can be studied wet or dry. An example of investigation done on the SM beamline is the characterization of magnetism of individual magnetosomes in magnetotactic bacteria [16]. Since the late 1960's, scientists have known that some bacteria make internal compasses by growing tiny magnetic crystals called magnetosomes. The bacteria use them to navigate, positioning themselves in environments most suited for their survival, with cells of the same species growing crystals of uniform size, structure and out of the same magnetic minerals.

\subsection{SAXS/WAXS capabilities}

The past decade has witnessed tremendous advances in instrumentation and interpretation of X-ray scattering results from macromolecules in solution. This makes possible the extraction of molecular shapes from analysis of scattering at very small diffraction angles (SAXS) [17, 18]. The increasing impact of SAXS is clearly evidenced by the ever-increasing number of publications describing applications of these techniques. A search of PubMed with the text 'small-angle X-ray scattering AND protein' showed the following number of hits: 367 (2010 to May 2011), 397 (2008-2009), 265 (2006-2007), 215 (2004$2005)$. In addition to the extremely useful information contained in the scattering at small angles, there is also a wealth of information that can be extracted from the scattering at wide angles (WAXS). These data provide more detailed information, corresponding to higher resolution and can describe conformational changes in proteins during their work cycle (time resolved experiments) [19]. SAXS and WAXS are also important tools for the characterization of drug delivery systems (nanomedicine) $[20,21]$.

At present there is no SAXS/WAXS beamline at the CLS. However, as a part of the Brockhouse X-Ray Diffraction and Scattering Sector, a material science SAXS/WAXS beamline is being built and potentially could be used by structural biologists. A dedicated Biological SAXS beamline is currently in the planning stage.

\subsection{Important Synergies}

At the University of Saskatchewan there are two Health Research Groups that are very closely associated with the Canadian Light Source: the Molecular Design Research Group (MDRG) and the CIHR Training Grant in Health Research Using Synchrotron Techniques (CIHRTHRUST). The CIHR-THRUST is a cross-disciplinary training and mentoring program intended for the development and use of synchrotron techniques to address key questions in Canadian health research. THRUST will train more than 50 of the next generation of Canadian synchrotron health researchers between 2009 and 2015. An example of work from the THRUST program is a publication on the influence of prion protein expression levels on local copper, iron and zinc content in the mouse brain [22]. The MDRG integrates the capabilities of the Canadian Light Source and the Saskatchewan Structural Sciences Centre into research focused on protein structure/function, signalling networks and drug development. An example of the collaborative work accomplished by the MDRG is the structural investigation of myo-inositol dehydrogenase from Bacillus subtilis [23].

\section{Conclusions}

The facilities for Structural Biology at the Canadian Light Source represent a Canadian national resource that is available to science and industry world-wide. Beamlines include state-of-the-art infrastructure and specialized capabilities, many of which are not available elsewhere. The current vision for Structural Biology at the Canadian Light Source is significantly enhanced by the synergies and collaborations between the users of the different beamlines and by the strength of the scientific personnel and trainees.

\section{Acknowledgements}

The authors would like to thank the whole CMCF staff, Dr. Chithra Karunakaran for providing information on X-ray Spectromicroscopy and Dr. Shaun Labiuk for careful reading of the manuscript and valuable suggestions. Work in the George laboratory is supported by a Canada Research Chair, Natural Sciences and Engineering Research Council of Canada, the Canadian Institutes of Health Research and the Saskatchewan Health Research Foundation. The Canadian Light Source is supported by the Natural Sciences and Engineering Research Council of Canada, the National Research Council of Canada, the Canadian Institutes of Health Research, the Province of Saskatchewan, Western Economic Diversification Canada, and the University of Saskatchewan.

\section{References}

[1] A. Kuller, W. Fleri, W.F. Bluhm, J.L. Smith, J. Westbrook, P.E. Bourne, Trends in Biochemical Sciences 23, 213 (2002). 
[2] P. Grochulski, M.N. Fodje, J. Gorin, S.L. Labiuk, R. Berg, J. Synchrotron Rad. 18, 681 (2011).

[3] M.N. Fodje, K. Janzen, R. Berg, G. Black, S. Labiuk, J. Gorin, P. Grochulski, J. Synchrotron Rad. 19, 274 (2012).

[4] M.N. Fodje, R. Berg, G. Black, P. Grochulski, K. Janzen, Automation of the Macromolecular Crystallography Beamlines at the Canadian Light Source, PCaPAC, Saskatoon (2010).

[5] W. Kabsch, Journal of Appl. Crystallogr.26, 795 (1993).

[6] P.R. Evans, Acta Cryst. D62, 72 (2006).

[7] N.4. Collaborative Computational Project, Acta Cryst. 50, 760 (1994).

[8] G.P. Bourenkov, A.N. Popov, Acta Cryst. D62, 58 (2006).

[9] K. Diederichs, Acta Cryst. D62, 96 (2006).

[10] M.L. Tonkin, M. Roques, M.H. Lamarque, M. Pugničre, D. Douguet, J. Crawford, M. Lebrun, M.J. Boulanger, Science 333, 463 (2011).

[11] M.A. DePristo, P.I. deBakker, T.L. Blundell, Structure 12, 831 (2004).

[12] M.J. Pushie, G.N. George, Coord. Chem. Rev. 255, 1055 (2011).

[13] B.F.G. Popescu, M.J. George, U. Bergmann, A.V. Garachtchenko, M.E. Kelly, R.P.E. McCrea, K. Lüning, R.M. Devon, G.N. George, A.D. Hanson, S.M. Harder, L.D. Chapman, I.J. Pickering, H. Nichol, Phys. Med. Biol. 54, 651 (2009).

[14] L. Zhang, J. Lichtmannegger, K.H. Summer, S. Webb, I.J. Pickering, G.N. George, Biochemistry 48, 891 (2009).
[15] K.V. Kaznatcheev, C.Ch. Karunakaran, U.D. Lanke, S.G. Urquhart, M. Obst, A. Hitchcock, Nucl. Inst. Meth. 582, 96 (2007).

[16] K.P. Lam, A.P. Hitchcock, M. Obst, J.R. Lawrence, G.D.W. Swerhone, G.G. Leppard, T. Tyliszczak, C. Karunakaran, J. Wang, K. Kaznatcheev, D.A. Bazylinski, U. Lins, Chemical Geology 270, 110 (2010).

[17] G.L Hura, L.A. Menon, M. Hammel, R. Rambo, L.F. Poole II, S.E. Tsutakawa, F. Jenney Jr, S. Classen, K. Frankel, R. Hopkins, S. Yang, J. Scott, B. Dillard, M. Adams, J.A. Tainer, Nature Methods 6, 606 (2009).

[18] T.D. Grant, J.R. Luft, J.R. Wolfley, H. Tsuruta, A. Martel, G.T. Montelione, E. Snell, Biopolymers 95 , 517 (2011)

[19] M. Cammarata, M. Levantino, F. Schotte, P.A. Anfinrud, F. Ewald, J. Choi, A. Cupane, M. Wulff, H. Ihee, Nature Methods 5, 881 (2008).

[20] C. Leal, N.F. Bouxsein, K.K. Ewert, C.R. Safinya, J. Am. Chem. Soc 132, 16841 (2010).

[21] Y. Dong, B.J. Boyd, Int. J. Pharm. 417, 101 (2011).

[22] M.J. Pushie, I.J. Pickering, G.R. Martin, S. Tsutsui, F.R. Jirik, G.N. George, Metallomics 3, 206 (2011).

[23] K.E. van Straaten, H. Zheng, D.R. Sanders, D. Palmer, Biochem. J. 432, 237 (2010). 